\title{
Case report of primitive neuroectodermal tumor with an unknown primary presenting with multiple lung metastasis
}

\author{
Jack Mizelle ${ }^{1}$, Jing Yang ${ }^{2}$, Eric Burks ${ }^{2}$, Virginia Litle ${ }^{3}$, Kei Suzuki ${ }^{3}$ \\ ${ }^{1}$ Boston University School of Medicine, Boston, MA, USA; ${ }^{2}$ Department of Pathology, ${ }^{3}$ Department of Surgery, Division of Thoracic Surgery, \\ Boston Medical Center, Boston, MA, USA \\ Correspondence to: Kei Suzuki, MD. Department of Surgery, Division of Thoracic Surgery, Boston Medical Center, Boston, MA, USA. \\ Email: Kei.suzuki@bmc.org.
}

\begin{abstract}
Ewing sarcoma or peripheral primitive neuroectodermal tumor (ES or pPNET) is a very aggressive cancer affecting children and young adults, with a five-year survival of $70-80 \%$ for localized disease and $30 \%$ for metastatic disease. ES can originate in any bone, but is most commonly reported in the lower extremities. To date, no cases of metastatic ES have been reported with an unknown primary in a patient over 45 years of age. We herein report the first documented case of metastatic ES to the lung with an unknown primary. Early diagnosis of rapidly invasive cancers such as ES is critical for effective treatment, which requires a multiple course combined chemotherapy regimen. The present case reports an atypical presentation of metastatic ES with bilateral involvement of the lungs and an unknown primary. We were able to definitively diagnose our patient with ES instead of other similar diseases such as small blue round cell tumor through immunohistochemical markers CD99 and NKX2.2, and began appropriate treatment.
\end{abstract}

Keywords: Ewing sarcoma (ES); metastasis; unknown primary

Received: 01 September 2019; Accepted: 18 September 2019; Published: 14 October 2019.

doi: $10.21037 /$ ccts.2019.09.03

View this article at: http://dx.doi.org/10.21037/ccts.2019.09.03

\section{Introduction}

Ewing sarcoma (ES) is a malignant soft tissue tumor derived from small undifferentiated neuroectodermal cells. They are the second most common bone tumor in children and young adults with approximately 225 new cases diagnosed each year in patients under the age of 20 years old in North America (1). The typical presentation includes locoregional pain with a primary lesion commonly found in the pelvic bones, long bones of the lower extremities, or bones of the chest wall. Overall prognosis is generally good for localized disease with the advancement of multi agentbased chemotherapy. However, approximately a quarter of patients presenting with metastases at diagnosis are resistant to intensive therapy and have poor prognosis (2). Herein, we report a rare case of metastatic ES with an unknown primary in a 49-year-old patient.

\section{Case presentation}

A 49-year-old previously healthy man presented with right neck pain, odynophagia, dysphagia, and fever of $38.3{ }^{\circ} \mathrm{C}$ and was transferred to our hospital. Vitals signs were stable except mild tachycardia at 100 . Laboratory examination indicated that the white blood cell count and neutrophil levels were increased (WBC $15.2 \mathrm{~g} / \mathrm{dL}$; Poly 89). Initial treatment consisted of intravenous clindamycin and vancomycin.

Chest computed tomography (CT) revealed a hypopharyngeal phlegmon/abscess involving the inferior right tonsil and supraglottic airway, as well as enlarged cervical lymph nodes and incidentally found multiple wellcircumscribed bilateral pulmonary nodules, with the largest being $3.5 \mathrm{~cm}$ (Figure 1). Percutaneous biopsy was performed but did not provide sufficient tissue for diagnosis. During 


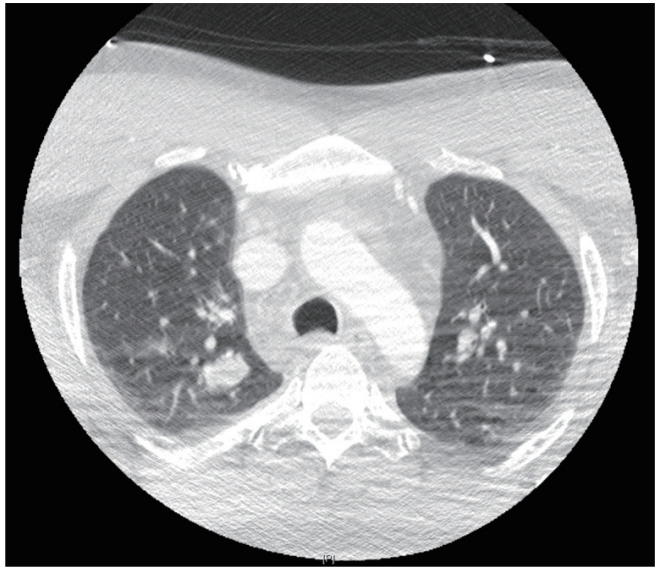

Figure 1 Computed tomography (CT) scan showing bilateral metastatic lung nodules at the level of aortic arch. the same admission, patient underwent an uncomplicated flexible nasolaryngoscopy in order to drain the right peritonsillar abscess.

As we did not have tissue diagnosis of the lung nodules, right lower lobe wedge biopsy was performed by videoassisted thoracoscopic surgery (VATS). The hematoxylin and eosin stained section showed a small round blue cell neoplasm with necrosis, suggestive of an undifferentiated small cell neoplasm. On immunohistochemistry studies, the tumor cells were positive for CD99 and vimentin, and negative for AE1:3, synaptophysin, chromogranin, CD56, CD34, and LCA, with a high proliferation rate, indicated by MIB-1 (Figure 2). Based on these findings, the differential at this point included ES and primitive neuroectodermal tumor (PNET) like small round blue cell tumor. Further
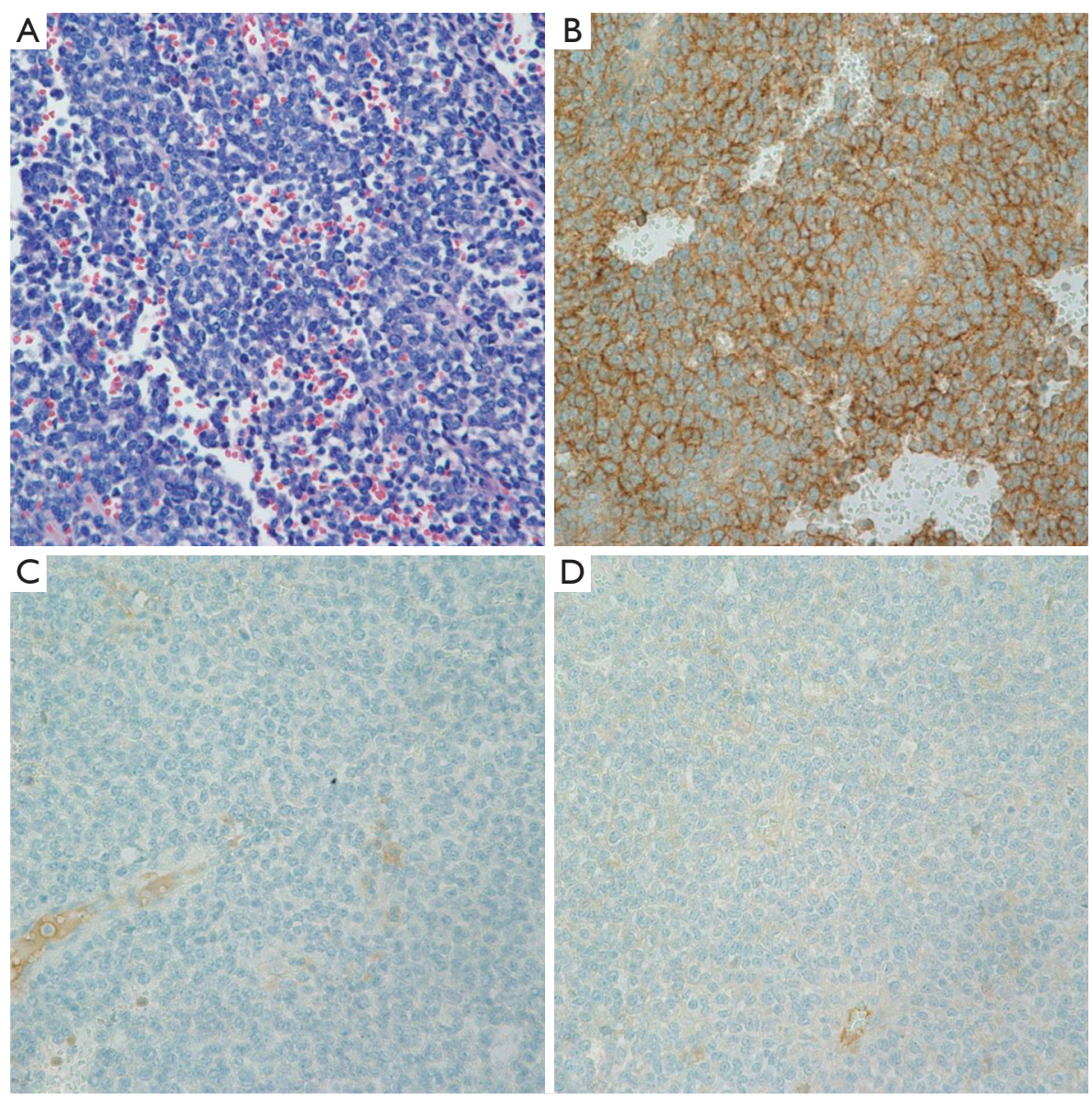

Figure 2 Right lung wedge biopsy revealed a small round blue cell neoplasm. The tumor cells were small round with vacuolated cytoplasm and uniform nuclei (A: $\times 400)$. The tumor cells showed strong membrane positivity for CD99 (B: ×400), and were negative for synaptophysin and chromogranin (C and D: $\times 400)$. 

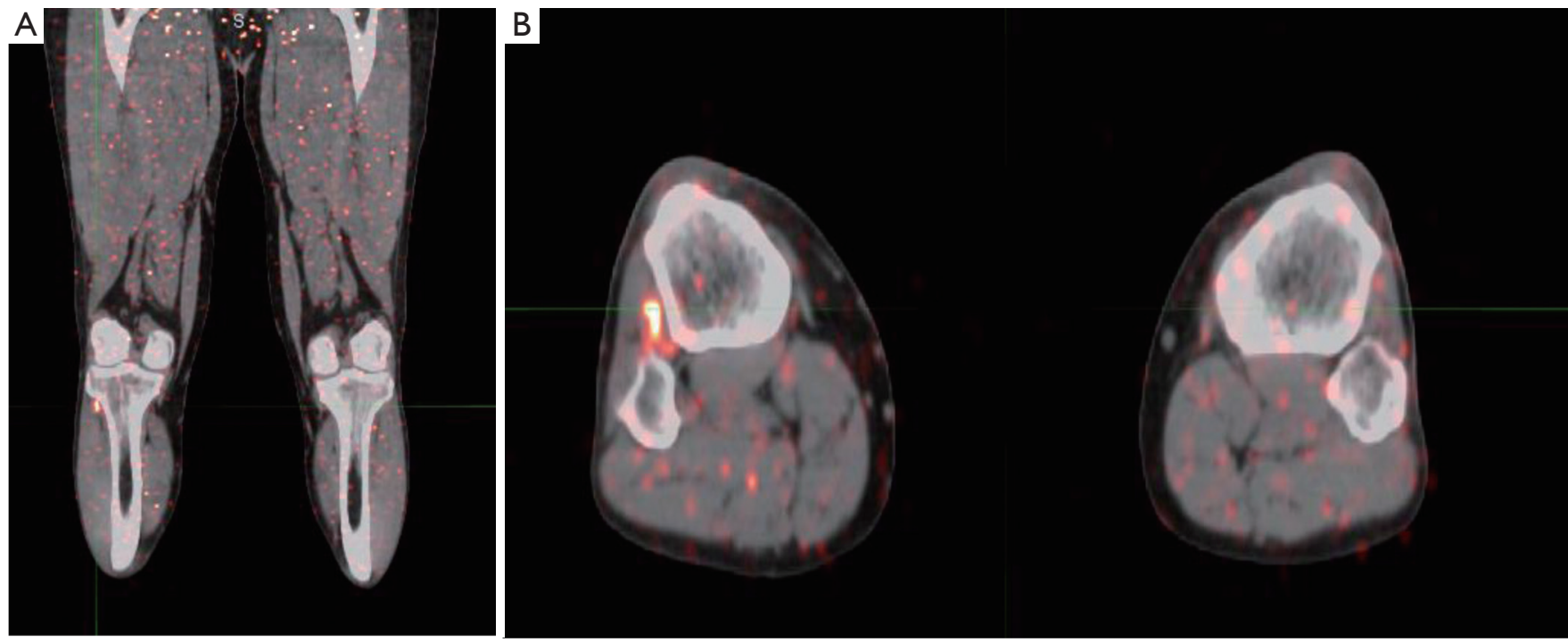

Figure 3 Gallium 68 dotatate scan showing (A) coronal (B) axial images of the lower knees corresponding with a region of focal radiotracer uptake at the interosseous membrane between the level of the right tibia.

Table 1 Timeline of relevant clinical history

Dates of major events

March 11, 2017: incidental finding on chest X-ray of bilateral pulmonary nodules with same finding on follow up CT

March 14, 2017: IR guided biopsy of lung nodules suggested small blue round cells

March 28, 2017: initial diagnosis of Ewing sarcoma from pathology review of surgical lung wedge specimen showing metastatic Ewing's sarcoma with positive CD 99, NKX2.2, and FISH for EWSR1 rearrangement

April 21, 2017: cancer staged by PET CT showing lung metastasis with no clear primary

May 2 to July 24: systemic therapy

CT, computed tomography.

review at an outside institution showed poorly differentiated round cell malignant neoplasm that was NKX2.2 positive. Fluorescence in situ hybridization (FISH) showed $96 \%$ tumor cells had EWSR1 rearrangement, consistent with metastatic ES.

A PET-CT scan was performed for staging of neuroendocrine tumor as part of initial treatment plan, which showed lung metastasis with no clear primary. A follow-up bone scan showed no dominant primary neuroendocrine lesion. Interestingly, the bone scan noticed an ovoid $1.5 \mathrm{~cm} \times 0.9 \mathrm{~cm}$ nodule positioned in the interosseous membrane between the proximal right tibia and fibula, with corresponding uptake on PET-CT (Figure 3). To explore the possibility of an undetected primary in this region, a MRI scan was performed and found a $1.5 \mathrm{~cm} \times$ $0.9 \mathrm{~cm} \times 1.3 \mathrm{~cm}$ (anterior-posterior $\times$ transverse $\times$ coronal $)$ ovoid lesion with focal radiotracer uptake, demonstrating intermediate internal enhancement after contrast.

The patient received a total of 14 cycles of chemotherapy composing of vincristine, doxorubicin, and cyclophosphamide, alternating with ifosfamide and etoposide. After the $6^{\text {th }}$ cycle, a CT chest abdomen and pelvis, comparing to initial PET/CT, found the two largest nodules from prior CT decreased slightly in size, with several other nodules appearing unchanged. For relevant dates of clinical history and treatment see Table 1.

\section{Discussion}

ES family tumors include ES of the bone and PNET. Central primitive neuroectodermal tumors (CPNET) were first characterized in 1997 (3). By 1985, ES was also found 
to originate from the neuroectoderm, and was termed as pPNET. Both ES and pPNET share similar chromosomal translocation, suggesting they both originate through mesenchymal differentiation of primitive neural stem cells (4).

Sarcomas in general are very rare, with about 12-13,000 cases in the United States per year, which represents approximately less than $1 \%$ of adult malignancies. There are approximately 50 to 100 different subtypes of soft tissue sarcomas. ES particularly occurs in children and adolescents. Adult ES is very rare with fewer than $5 \%$ of cases in patients over 40 years old (5). Approximately 25\% of ES patients have

detectable metastasis at diagnosis, with the most common sites for metastasis being lungs (50\%), bone (25\%), or bone marrow (20\%) (5). Rare cases have also been documented affecting the spinal column.

$\mathrm{ES}$ is aggressive, with microscopic metastasis being common and presumed at diagnosis. For about a quarter of patients with ES, surgery alone is insufficient to treat this disease. Cure is often obtained with the use of multi-agent chemotherapy regimens due to the tumor's responsiveness to systemic chemotherapy and radiation. With appropriate systemic chemotherapy cure approaches $70 \%$ to $80 \%$ for patients with localized disease and $10 \%$ to $20 \%$ for patients with metastatic disease at diagnosis (6).

The present case is particularly interesting in that: (I) this was seen in adult onset and (II) patient presented with incidentally found lung metastasis with unknown primary. With wide involvement of both lungs, we expected to find a prominent intra or extraosseous primary. This is the first case report in the literature to document a case of metastatic ES with an unknown primary in a middle-aged man.

Interestingly, a bone scan and follow-up MRI localized a small ovoid lesion in the interosseous membrane at the level of the right tibia without involvement of the surrounding soft tissue and muscles. The possibility of a localized ES primary within the interosseous membrane is very low due to the poor environmental conditions for soft tissue tumors growing in the fibrotic interosseous membrane. The small ovoid lesion in the interosseous membrane may be a separate finding from ES. Other possibilities include a peripheral nerve sheath tumor like a schwannoma, atypical ganglion cyst of the proximal tibiofibular joint, metastatic soft tissue nodule or soft tissue sarcoma.

The present case reports an atypical presentation of metastatic ES with bilateral involvement of the lungs and an unknown primary. In comparison, few cases in the literature report interosseous involvement.
Notably, one case reported a $7 \mathrm{~cm}$ enhancing small blue round cell tumor associated with the interosseous membrane characterized by MRI. However, unlike the present case, their patient presented with pain and swelling in the leg, and a non-metastatic mass lying in the posterior calf with popliteus muscle involvement, extending through the interosseous membrane, and encasing both the tibial nerve and popliteal vessel (7).

\section{Conclusions}

Early recognition of the varying degrees of the clinical presentation of metastatic ES provides a substantially better prognosis, through the timely use of appropriate combined chemotherapy regimens. ES often involves early metastasis involving the pulmonary system. Despite an unclear primary, our patient presented with extensive metastatic involvement of both lungs, which clued us into considering the possibility of ES in our differential of patients. The present case was able to definitely diagnose our patient with ES instead of other similar diseases such as small blue round cell tumor through immunohistochemical markers CD99 and NKX2.2, and began appropriate treatment with multiagent therapy.

\section{Acknowledgments}

Funding: None.

\section{Footnote}

Conflicts of Interest: All authors have completed the ICMJE uniform disclosure form (available at http://dx.doi. org/10.21037/ccts.2019.09.03). The authors have no conflicts of interest to declare.

Ethical Statement: The authors are accountable for all aspects of the work in ensuring that questions related to the accuracy or integrity of any part of the work are appropriately investigated and resolved. Written informed consent was obtained from the patient for publication of this manuscript and any accompanying images.

Open Access Statement: This is an Open Access article distributed in accordance with the Creative Commons Attribution-NonCommercial-NoDerivs 4.0 International License (CC BY-NC-ND 4.0), which permits the noncommercial replication and distribution of the article with 
the strict proviso that no changes or edits are made and the original work is properly cited (including links to both the formal publication through the relevant DOI and the license). See: https://creativecommons.org/licenses/by-nc-nd/4.0/.

\section{References}

1. Esiashvili N, Goodman M, Marcus RB Jr. Changes in incidence and survival of Ewing sarcoma patients over the past 3 decades: Surveillance Epidemiology and End Results data. J Pediatr Hematol Oncol 2008;30:425-30.

2. Gaspar N, Hawkins DS, Dirksen U, et al. Ewing sarcoma: current management and future approaches through collaboration. J Clin Oncol 2015;33:3036-46.

3. Hart NH, Earle KM. Primitive neuroectodermal tumors

doi: $10.21037 /$ ccts.2019.09.03

Cite this article as: Mizelle J, Yang J, Burks E, Litle V, Suzuki $\mathrm{K}$. Case report of primitive neuroectodermal tumor with an unknown primary presenting with multiple lung metastasis. Curr Chall Thorac Surg 2019;1:15. of the brain in children. Cancer 1973;32:890-7.

4. Jaffe R, Santamaria M, Yunis EJ, et al. The neuroectodermal tumor of bone. Am J Surg Pathol 1984;8:885-98.

5. Balamuth NJ, Womer RB. Ewing's sarcoma. Lancet Oncol 2010;11:184-92.

6. Burdach S, Meyer-Bahlburg A, Laws HJ, et al. Highdose therapy for patients with primary multifocal and early relapsed Ewing's tumors: results of two consecutive regimens assessing the role of total-body irradiation. J Clin Oncol 2003;21:3072-8.

7. Pacheco M, Horsman DE, Hayes MM, et al. Small blue round cell tumor of the interosseous membrane bearing at $(2 ; 22)$ (q34; q12)/EWS-CREB1 translocation: a case report. Mol Cytogenet 2010;3:12. 